\title{
Effect of Magnetic Field on the Ionization Potential of the
} Atoms and Ions

\author{
Aibassov Erkin Zhakenovich", Yemelyanova Valentina, Tussupbayev Nessipbay, Shakieva Tatyana and \\ Yerzhanova Zhadyra \\ Research Institute of New Chemical Technologies and Materials, Kazakh National University Al-Farabi, Almaty 005012, Kazakhstan
}

\begin{abstract}
The authors found the effect of magnetic field on the ionization of atoms and ions and shown that the magnetic field affected the rate of ionization and electron emission at angle of $60^{\circ}, 120^{\circ}, 240^{\circ}$ and $300^{\circ}$. It is shown that the calculation must take into account the ionization potential of the magnetic field.
\end{abstract}

Key words: Magnetic field effect, ionization potential, rate of ionization, electron emission angle.

\section{Introduction}

The ionization potential of the atom - the minimum potential $\mathrm{U}$, which will be held in the electron accelerating electric field to acquire enough kinetic energy to ionize an atom. Ionization energy $E$ is the minimum energy that must be expended to remove an electron from an atom.

Ionization potential $U$ is closely related to the ionization energy ratio:

$$
E=U e
$$

where, $e$ is elementary charge. Since the electric and magnetic fields are interrelated, it was interesting to see the effect of the magnetic field on the ionization potential.

The importance of studying the effect of magnetic field on the ionization potential is not in doubt.

\section{Theory}

\subsection{Effect of Magnetic Field on the Rate of Ionization of Atoms}

In a magnetic field on the electron Lorentz force, resulting in extreme sub-barrier trajectory "twists" and is no longer one-dimensional [1-5].

\footnotetext{
*Corresponding author: Aibassov Yerkin Zhakenovich, professor, research fields: organic chemistry of $\mathrm{U}, \mathrm{Th}, \mathrm{As}, \mathrm{Sb}$, Bi. E-mail: erkin53@mail.ru.
}

It is necessary to solve the equation of motion

$$
\mathrm{r}^{* *}=-\mathrm{E}-1 / \mathrm{c}\left[\mathrm{r}^{*} \mathrm{H}\right]-\mathrm{Zr} / \mathrm{r}^{2}
$$

with a complex "time" $\mathrm{t}$ (in atomic units $\mathrm{c}=\alpha^{-1}$

= 137), what can be done analytically only at $\mathrm{Z}$

$=0$. Taking into account the boundary condition $\mathrm{s}$ for short-range potential:

$$
r\left(t_{0}\right)=0, r^{*}\left(t_{0}\right)=-k^{2}=-2 I, \operatorname{Im} r(t=0)=0
$$

it can be found (in the case of constant fields e and $\mathrm{H}$ ) this trajectory:

$$
\begin{gathered}
\mathrm{X}=\mathrm{iE} / \omega_{\mathrm{c}}{ }^{2}\left(\tau-\tau_{0} \sinh \tau / \sinh \tau_{0}\right) \sin \theta \\
\mathrm{Y}=\mathrm{E} / \omega_{\mathrm{c}}{ }^{2} \tau_{0} / \sinh \tau_{0}\left(\cosh \tau-\cosh \tau_{0}\right) \sin \theta \\
\mathrm{Z}=\mathrm{E} / \omega_{\mathrm{c}}{ }^{2}\left(\tau_{0}{ }^{2}-\tau^{2}\right) \cos \theta
\end{gathered}
$$

where, $I=k^{2} / 2$, the ionization potential of the atomic level, $\tau=i \omega_{\mathrm{c}} \mathrm{t}$, where $\omega_{\mathrm{c}}-$ cyclotron, or the Larmor frequency:

$$
\omega_{\mathrm{c}}=\mathrm{H} / \mathrm{c}
$$

directed along the $\mathrm{Y}$ axis $\mathrm{E}, \mathrm{X}$ axis perpendicular to the plane $(E, H), \theta-$ angle between these fields. Thus, when the magnetic field under the influence of the Lorentz forces is curved trajectory sub-barrier, the barrier width increases.

\subsection{Angular Distributions in an Elliptically Polarized Field \\ Coulomb interaction affects not only the total ionization probability, but also on the momentum distribution of electrons.}


The result of the Coulomb interaction is the effect of breach of the fourfold symmetry of the angular distributions of electrons in a field with elliptical polarization. The calculation of the angular distributions of electrons with energy $\mathrm{E}=7.9 \mathrm{eV}$ in the ionization of the ground state of the hydrogen atom field Ti: sapphire laser $\left(\mathrm{h} \omega=1.55 \mathrm{eV}, \mathrm{J}=10^{14}\right.$ $\mathrm{W} \cdot \mathrm{sm}^{-2}$, ellipticity $\rho=0.5$ ) showed that the directions of emission of electrons with the same values of the ionization probability is: $60^{\circ}, 120^{\circ}, 240^{\circ}$ and $300^{\circ}$, which corresponds to the Fermi surface (Fig. 1). For intermediate values of ellipticity $\rho, 0.2<\rho<0.8$, most likely the electron momentum is directed along the minor axis of the polarization ellipse. This is obvious when you consider that the electrons are produced at the output of the barrier, with zero velocity. Tunneling probability is a maximum at the moment when the electric field reaches its maximum. When this minimal vector potential magnitude and directed along the minor axis of the ellipse of polarization. Just sent and momentum $\mathrm{p}=\mathrm{v}-\mathrm{A} / \mathrm{c}$.

\section{Results and Discussion}

\subsection{Lorentz Ionization of Atoms and Ions}

If an atom or ion enters in a constant magnetic field
$\mathrm{H}$, then rest in their systems appears due Lorentz electric field $\mathrm{E}_{0}$, which can lead to ionization. This process is called the Lorentz ionization. Limited to cases in which the velocity of the atom $\mathrm{v}$ perpendicular to the magnetic field $\mathrm{H}$. Then fields acting in the rest frame of the atom, are of the form:

$$
\mathrm{E}_{\mathrm{o}}=v / \sqrt{1-v^{2}} \mathrm{H}, \mathrm{H}_{\mathrm{o}}=1 / 1-v^{2} \mathrm{H}, \mathrm{E}_{\mathrm{o}} \perp \mathrm{H}_{\mathrm{o}}
$$

and the parameter is equal to the adiabatic:

$$
\gamma_{\mathrm{L}}=\mathrm{kH}_{0} / \mathrm{cE}_{0}=\mathrm{k} / \mathrm{v}
$$

where, the velocity $\mathrm{v}$ is expressed in atomic units (1 a.e. $=\mathrm{e}^{2} / \mathrm{h}=2.19 \times 10^{8} \mathrm{sm} \cdot \mathrm{s}^{-1} \approx 0.007 \mathrm{~s}$ ). For the ground state of neutral atoms parameter $\mathrm{k}$ is close to unity.

Speed Lorentz ionization has the form:

$$
\mathrm{w}_{\mathrm{L}}=\left(1-\mathrm{v}^{2}\right)^{1 / 2} \mathrm{~S} w\left(\mathrm{E}_{0}\right)
$$

where, $w\left(E_{0}\right)$ - the rate of ionization of the atom under the influence of an electric field only $E_{0}$, $\mathrm{S}$-stabilization factor, taking into account the suppression of the decay of the atomic level magnetic field. For fast ( $\mathrm{v}>10 \mathrm{k}$ ) particles $\mathrm{S} \rightarrow 1$, i.e., the ionization of the atomic level occurs almost at the same rate as in the pure electric field $E_{0}$ (factor $\sqrt{1-v^{2}}$ takes into account the effect of time dilation for a moving atom).

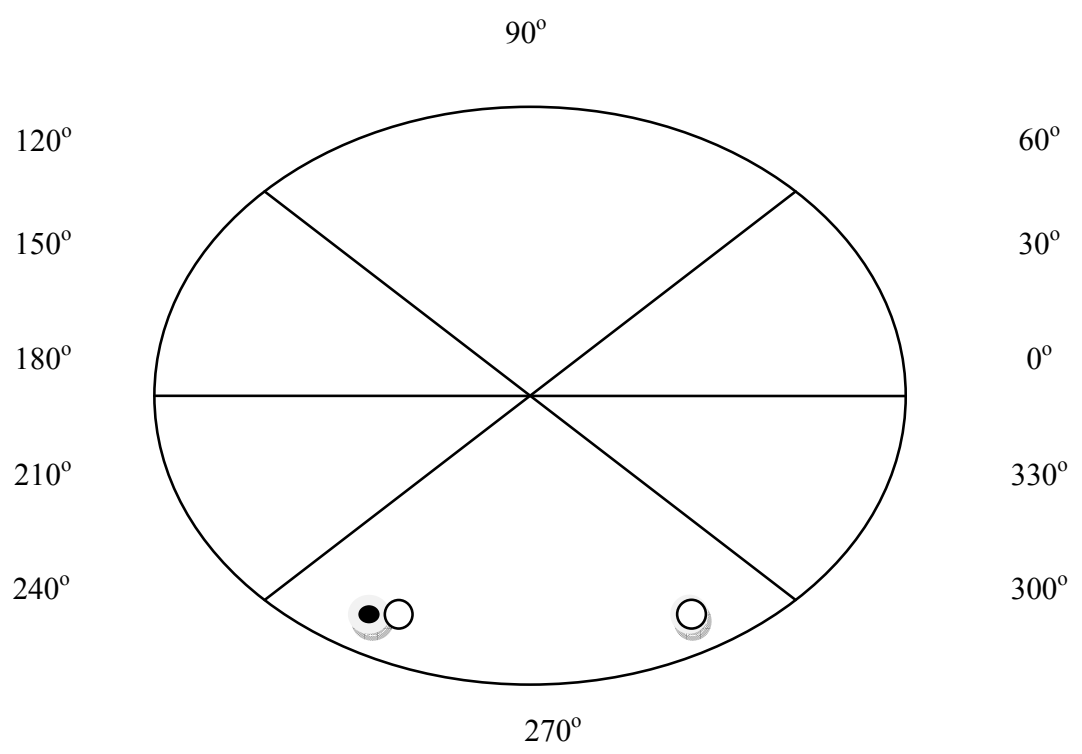

Fig. 1 The angular distribution of electrons with energy $E=7.9 \mathrm{eV}$ in the ionization of the ground state of the hydrogen atom by the laser field $\left(\mathrm{h} \omega=1.55 \mathrm{eV}, \mathrm{J}=1014 \mathrm{~W} \cdot \mathrm{sm}^{-2}\right.$, ellipticity $\left.\rho=0.5\right)$. 
Table 1 Probability of Lorentz ionization of the hydrogen atom.

\begin{tabular}{llll}
\hline$v$ (a.u.) & $\mathrm{E}_{0}$ (a.e.) & $\mathrm{S}$ & $\mathrm{W}_{\mathrm{L}}$ (a.u.) \\
\hline 0.5 & $5.32(-3)$ & $3.7(-9)$ & 0 \\
1.0 & $1.06(-2)$ & 0.119 & $1.03(-9)$ \\
1.25 & $1.33(-2)$ & 0.345 & $6.41(-4)$ \\
1.67 & $1.77(-2)$ & 0.645 & 2.40 \\
2.0 & $2.13(-2)$ & 0.779 & $1.55(5)$ \\
2.5 & $2.66(-2)$ & 0.882 & $5.56(7)$ \\
5.0 & $5.32(-2)$ & 0.984 & $6.24(12)$ \\
10 & 0.107 & 0.998 & $7.73(14)$ \\
\hline
\end{tabular}

where, $v=1$ a.u. corresponding to a velocity of the atom $2.19 \times 10^{8} \mathrm{sm} \cdot \mathrm{s}^{-1} \approx 0.007 \mathrm{~s} . \mathrm{H}=25 \mathrm{MHz}-$ maximum magnetic field, $\mathrm{E}_{0}-$ the electric field acting in the rest frame of the atom.

If $\mathrm{H}=1 \mathrm{MHz}$ atom stable, since the electric field $\mathrm{E}_{0}$ is too low. At $\mathrm{H}=25 \mathrm{MHz}$ in the range of velocities $\mathrm{V}$ $=0.5$ a.u. to $\mathrm{v}=10$ a.u. The situation varies from complete stabilization of the atom to its ionization in a time comparable to the atomic time $\tau_{\mathrm{a}}=\mathrm{h}^{3} /\left(\mathrm{me}^{4}\right)\left(\mathrm{w}_{\mathrm{a}}\right.$ $\left.=1 / \tau_{\mathrm{a}}=4.13 \times 10^{16} \mathrm{~s}^{-1}\right)$. In the case of negative ions with a low binding energy dependence of the ionization probability of $\mathrm{v}$ and $\mathrm{H}$ has the same form as for the neutral atoms [6-9].

Table 1 shows the probability of Lorentz ionization of the hydrogen atom.

\subsection{The General Expression for the Ionization}

Probability in the Relativistic Case

Consider the ionization of atomic level with energy $\varepsilon_{0}$ in the Coulomb potential of a "tail", $\mathrm{U}_{\mathrm{C}}=-\mathrm{Z} \alpha / \mathrm{r}$. At large distances from the nucleus wave function corresponding to the quantum numbers $\mathrm{j}, \mathrm{l}, \mathrm{M}$, is given by:

$$
\begin{gathered}
\Psi_{\mathrm{jlM}}{ }^{(0)}\left(\mathrm{r}, \mathrm{t}, \varepsilon_{0}\right)=\mathrm{C}_{\mathrm{jlM}}{ }^{3 / 2}\left(\sqrt{1+\varepsilon_{0}}\right)\left(\mathrm{i} \sqrt{1+\varepsilon_{0}} \quad \text { on }\right) \times \mathrm{R}_{0}(\mathrm{r}) \\
\Omega_{\mathrm{j} 1 \mathrm{M}}(\mathrm{n}) \exp \left(\mathrm{i} \varepsilon_{0} \mathrm{t}\right)
\end{gathered}
$$

where, $\lambda_{0}=\sqrt{1+\varepsilon_{0}^{2}}, \sigma-$ Pauli matrices, $n=r / r=1$,

$$
\begin{aligned}
& \Omega_{\mathrm{jlM}}(\mathrm{n})-\text { spherical spinors, } \mathrm{C}_{\mathrm{jlM}}-\text { dimensionless } \\
& \text { asymptotic coefficient, } \\
& \mathrm{R}_{0}(\mathrm{r})=\left(\lambda_{0} \mathrm{r}\right)^{v-1} \exp \left(\lambda_{0} \mathrm{r}\right), \mathrm{r}>>1 / \lambda_{0}
\end{aligned}
$$

where, $v=\mathrm{Ze}^{2} \varepsilon_{0} / \lambda_{0}-$ relativistic analog of the effective principal quantum number. For the ground state of hydrogen-like ion with nuclear charge $Z$, the authors have $\mathrm{j}=\mathrm{M}=1 / 2, \mathrm{l}=0$ and

$$
\begin{gathered}
\varepsilon_{0}=\sqrt{1-(\alpha Z)^{2}} \\
\lambda_{0}=\alpha Z, \mathrm{C}_{1 / 2,0,-1 / 2}^{2}=2^{2} \varepsilon_{0} / \Gamma\left(2 \varepsilon_{0}+1\right)
\end{gathered}
$$

where, $\Gamma(\ldots)$-Euler gamma function. Currently known atoms correspond to values $Z<137$ and $\varepsilon_{0}>0$, in this case, the approximation of a point charge of the nucleus.

The ionization probability is determined by the truncated part of the action $\mathrm{W}$, calculated along the extreme sub-barrier trajectory:

$$
\mathrm{w}_{\mathrm{R}}\left(\varepsilon_{0} ; \mathrm{jlM}\right)=\mathrm{w}_{0}\left|\mathrm{C}_{\mathrm{jlM}}\right|^{2} \mathrm{P}_{\mathrm{jlM}} \mathrm{Q} \exp (-2 \mathrm{Im} \mathrm{W})
$$
where, Q-Coulomb factor, pre-exponential factor $\mathrm{P}_{\mathrm{jlM}}$ includes the spin factor,

$$
\begin{array}{r}
\mathrm{W}=\int\left(\mathrm{L}-\varepsilon_{0}\right) \mathrm{dt}=\mathrm{S}-\varepsilon_{0} \mathrm{t} \\
\mathrm{w}_{0}=\mathrm{mc}^{2} / \mathrm{h}=0.776 \times 10^{21} \mathrm{~s}^{-1}
\end{array}
$$

\section{Conclusions}

The authors have examined the effect of a magnetic field on the ionization of atoms and ions. It is shown that the magnetic field affects the rate of ionization and electron emission angle.

The results obtained allow people to explain many of the questions on the rate of ionization and electron emission angle strictly under a certain degree.

\section{Acknowledgments}

The authors would like to thank Lynn C. Francesconi (Hunter College CUNY), Ruben M. Savizky (Columbia University, New York), Peter C. Burns (Notre Dame 
University, Indiana) and Chistopher L. Cahill (George Washington University) for discussion of the results.

\section{References}

[1] Hutchings, G. J., Heneghan, C. S., Hudson, I. D., and Taylor, S. H. 1996. "Uranium-Oxide-Based Catalysts for the Destruction of Volatile Chloro-Organic Compounds." Nature 384: 341-3.

[2] Grasselli, R. K., and Callahan, J. L. 1969. "Structure-Catalytic Efficiency Relationships in U-Sb Oxide Acrylonitrile Synthesis Catalysts." J. Catal. 14: 93-103.

[3] Corberan, V. C., Kremenic, G., and Tejuca, G. 1988. "Acidity and Oxidation Activity of $\mathrm{MoO}_{3}-\mathrm{UO}_{3} / \mathrm{SiO}_{2}$ Catalysts." React. Kinet. Catal. Lett. 36: 235-40.

[4] Gordeeva, L. G., Aristov, Y. I., Moroz, E. M., Rudina, N. A., Zaikovskii, V. I., and Tanashev, Y. Y. et al. 1995. "Preparation and Study of Porous Uranium Oxides as Supports for New Catalysts of Steam Reforming of Methane.” J. Nucl. Mater. 218: 202-9.

[5] Karnakov B. M., Mur, V. D., Popruzhenko, S. V., and
Popov, V. S. 2015. "Modern Development of the Theory of Nonlinear Ionization of Atoms and Ions, Zh." Phys. Sceince N1: 3-34.

[6] Aibassov, E. Z., and Umirkulova, Z. S. 2012. "Getting the Uranium Catalyst Based on Natural Zeolite Deposits Chankanayskogo." In Proceedings of Symposium "Fronties of Organometallic Chemistry, Saint-Petersburg, Voronkov, M. G., Ed., Saint-Petersburg: Saint-Petersburg University, Russia.

[7] Aibassov, E. Z., and Aibassova, S. M. 2011. "The New Reactions of Arsine." Introduction to Organic Chemistry of Arsenic, Actinides, Lanthanides, $\mathrm{Os}^{187}$ and Re; edited by Aibassov E. Z. Vol. 1. New York: Gitel Publishing House, Inc., 100-2.

[8] Aibassov, Y. Z., and Yemelyanova, V. S. 2014. "Effect of Magnetic Field on the Catalysis of Ligands in Non-aqueous Solutions." Journal of Chemical Engineering and Chemistry Research 1 (2): 132-7.

[9] Aibassov, Y. Z., and Yemelyanova, V. S. 2014. "Magnetism in Chemistry". Magnetic and Relativistic Effects in Catalysis and Uranium Catalysts. Almaty: Infiniline Publishing House, Inc., 184-6. 